\title{
Vibration Control of Double-Beam System with Multiple Smart Damping Members
}

\author{
Dominik Pisarski, ${ }^{1}$ Tomasz Szmidt, ${ }^{1}$ Czesław I. Bajer, ${ }^{1}$ \\ Bartłomiej Dyniewicz, ${ }^{1}$ and Jacek M. Bajkowski ${ }^{2}$ \\ ${ }^{1}$ Institute of Fundamental Technological Research (IPPT), Polish Academy of Sciences, Pawinskiego 5b, 02-106 Warsaw, Poland \\ ${ }^{2}$ Faculty of Production Engineering Warsaw University of Technology, Narbutta 85, 02-524 Warsaw, Poland \\ Correspondence should be addressed to Dominik Pisarski; dpisar@ippt.pan.pl
}

Received 27 May 2016; Revised 2 September 2016; Accepted 19 September 2016

Academic Editor: Andras Szekrenyes

Copyright (C) 2016 Dominik Pisarski et al. This is an open access article distributed under the Creative Commons Attribution License, which permits unrestricted use, distribution, and reproduction in any medium, provided the original work is properly cited.

\begin{abstract}
A control method to stabilize vibration of a double cantilever system with a set of smart damping blocks is designed and numerically evaluated. The externally controlled magnetorheological sheared elastomer damping block is considered, but other smart materials can be used as well. The robust bang-bang control law for stabilization the bilinear system is elaborated. The key feature of the closed loop controller is the efficiency for different types of initial excitement. By employing the finite element model, the performance of the controller is validated for strong wind blow load and concentrated impact excitement of the particular point of one of the beams. For each of the excitations, the closed loop control outperforms the optimal passive damping case by over $27 \%$ for the considered energy metric.
\end{abstract}

\section{Introduction}

Passive damping solutions are appreciated for simplicity and robust performance, but they may be insufficient when resistance to incidental extreme loads is considered. When introducing additional damping to improve the system, it is pragmatic to incorporate some vibration control possibility. Semiactive techniques allow adjusting damping parameters of the system while being easy to operate without high power consumption. Application of smart materials for adaptivepassive or semiactive operation regimes is recently in favour of scientists. In these cases, damping forces are developed due to the variation of the system motion and not due to direct application of the control force as in fully active solutions. The system requires less power to operate and is less susceptible to instability and malfunctions [1].

In this paper, the authors focus on the application of control regime, which is based on mistuning the system motion according to a state-feedback control law. The strategy aims at absorbing energy from one characteristic mode and releasing it at a particular instant of the vibration, increasing higher modes of the double-beam system. A stepwise parametric modification of the system allows achieving the desired level of vibration damping and is often referred to as the switching times control [2], adaptive elasticity [3], or prestress-accumulation-release [4]. Usually, the switching operates between two extreme control values, as in the bangbang type of control, or between two states of the actuator, as in an on-off logic $[5,6]$.

When beam systems are considered, different smart materials may be employed to perform semiactive damping strategy. The switchable stiffness is frequently obtained with piezoelectric patches. Ramaratnam and Jalili [7] applied switched piezoelectrics to stabilize flexible beam with a moving base, intended for robotic application. The phase transformation of shape memory alloys and polymers was used in [8] for morphing of aircraft wings. Ostachowicz et al. [9] used shape memory to stabilize smart beams. In [3], the authors varied the flexural bending stiffness of a multilayered beam with sheared polymer layers. The shear 


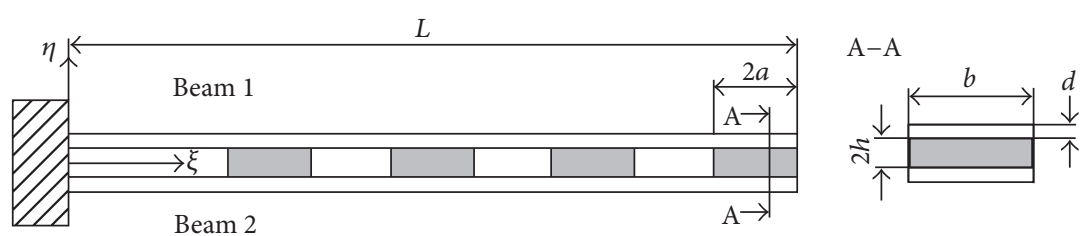

FIGURE 1: Double-beam structure with emebedded intelligent damping elements.

modulus switching of the polymer layer was obtained by heating it with ultrathin electric blankets. Recently, the present authors in $[10,11]$ proposed a pneumatic damping member based on switching the jamming transition of the granular material. Interesting practical examples of robust switched control based on a variable stiffness was applied to civil structures by means of on-off hydraulic devices [1214]. Winthrop et al. in [15] provided a good review of the most notable solutions based on the periodic switching of the system parameters.

The paper [16] devoted to the theoretical and experimental investigation of free vibrations of a layered beam with a single magnetorheological elastomer member proved the concept of switch stiffness to be effective, giving $20 \%$ improvement of the damping performance compared to the passive system. The research advances motivated us to study the performance of a more complex system, where several smart blocks are collaborating to counteract the vibrations introduced by distributed or impact loads, introducing higher structural modes.

The contribution of the work lies in the derivation, verification, and characterization of semiactive control strategy for independently operated smart blocks coupling two beams. The control logic is proposed, and theoretical effectiveness is demonstrated in numerical simulations for multidegree of freedom structure with multiple variable-stiffness members. As far as different types of smart materials can be used as actuators, we do not suggest any particular type to be controlled with the derived algorithm.

\section{Mathematical and Numerical Model}

The double-beam structure to be investigated consists of two elastic face sheets coupled by several viscoelastic smart damping elements (see Figure 1). Both beams have length $L$, width $b$, thickness $d$, and mass per unit length $\rho S$ and are mounted in a clamped support. We are going to investigate vibrations of the structure in the plane of symmetry $\xi-\eta$. The displacements of beams from their equilibria in $\eta$ direction are denoted by $w_{1,2}(\xi, t)$. The beams are slender and their deflections are small, so the linear Bernoulli-Euler beam theory is valid. The external damping of the beams is neglected. The effect of gravity, which acts perpendicularly to the plane of vibrations, is not taken into account either, since the equilibrium state is taken as a neutral one.

We consider $m$ identical damping blocks of rectangular shape $2 a \times b \times 2 h$. Their centers are located at distances $\xi_{1}, \ldots, \xi_{m}$ from the support. The rheological parameters of these elements, namely, the storage and loss moduli, are controllable by means of external devices embedded in the structure or placed nearby. The masses of the damping blocks as well as control devices are assumed to be negligible compared to the mass of the beams. Alternatively, they might be incorporated into the mass of the beams, as the damping blocks are stuck uniformly across the length of the structure.

To be more specific, we are going to consider a magnetorheological elastomer as the material of the damping blocks. Exposed to a magnetic field, the MRE increases its storage and loss moduli. Still, other smart materials, for instance, vacuum packed granules, can be applied as well. The overall elastic and dissipative characteristics of the structure are to a large extent determined by the rheological parameters of the damping elements. Thus, modification of these parameters allows controlling the system. Note that the control changes both parameters, that is, the storage and the loss modulus.

Every element is assumed to be made of a Kelvin-Voigt material. The shear stress-strain relation is given by

$$
\tau=h_{1} \varphi+h_{2} \dot{\varphi}
$$

where $\tau, \varphi$ are the shear stress and angular deformation, $h_{1}=h_{1}(u, \omega)=G^{\prime}(u, \omega)$ is the storage modulus, $h_{2}=$ $h_{2}(u, \omega)=G^{\prime \prime}(u, \omega) / \omega$ is the loss modulus divided by the angular frequency of vibrations $\omega$, and $u$ is the magnitude of the applied magnetic field. Although the values of $G^{\prime}$ and $G^{\prime \prime}$ depend on the frequency of vibrations, a typical change of storage or loss modulus due to the applied magnetic field yields only a slight change of this frequency. The values of $G^{\prime}$ and $G^{\prime \prime}$ are nearly constant within such a narrow range of $\omega$, so we may treat the values of both rheological parameters as independent of it. The relations between these parameters and the magnetic field are generally nonlinear. However, the external devices are able to control these parameters in a limited range only, where linear approximations are justified. Additionally, for the purpose of control design, we assume these relations to be monotonically growing.

The damping blocks undergo shear deformations when the beams are deflected. This yields bending moments as well as axial forces acting on both beams. Moreover, relative motion of the beams generates transverse forces. To develop the formulas for all these forces and moments, it turned out to be convenient to use the truss model proposed in [17]. Having assumed that the blocks are not too long $(2 a \ll L)$ and treating the parts of the beams over the damping blocks as rigid bodies, we may reduce the forces to the pole, which is the point over the center of the block (see Figure 2) where the two-link diagonal truss replaced the damping block attached at tip $(j=m)$. 


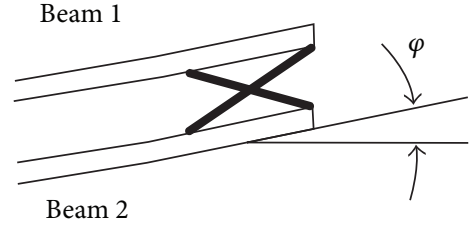

(a)

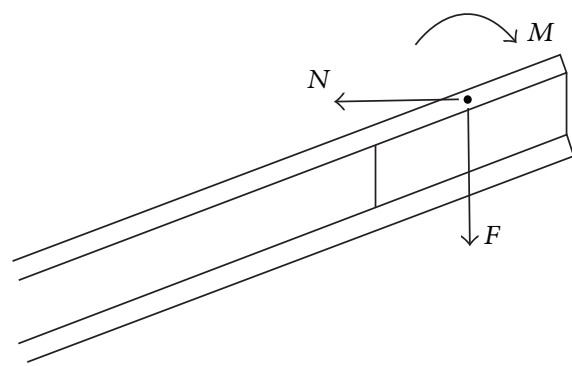

(b)

FIGURE 2: Truss model of the damping block (a) and forces generated by the damping block reduced to the pole (b).

Let us present the formulas for the bending moments and forces acting on Beam 1. Assume that the angle of deflection of both beams at point $\xi_{j}$ is the same and equal to $\varphi_{j}$, and the relative displacement of the beams at $\xi_{j}$ amounts to $\Delta w_{j}=$ $w_{1}\left(\xi_{j}, t\right)-w_{2}\left(\xi_{j}, t\right)$. Then, we come up with the following expressions for bending moments $M_{j}$ :

$$
M_{j}=-2 a b h\left(h_{1} \varphi_{j}+h_{2} \dot{\varphi}_{j}\right),
$$

transverse forces $F_{j}$ :

$$
F_{j}=-\frac{b h}{a}\left(h_{1} \Delta w_{j}+h_{2} \Delta \dot{w}_{j}\right)
$$

and axial forces $N_{j}$ :

$$
N_{j}=-2 a b\left(h_{1} \varphi_{j}+h_{2} \dot{\varphi}_{j}\right) .
$$

The influence of the axial forces appears to be of minor importance and it will be disregarded in the study. This is mainly because the axial forces act in the opposite directions; that is, when Beam 1 is compressed, then Beam 2 is stretched, and partly because these forces are too small to disturb the dynamics of the rigid face sheets.
The dynamics of the system are governed by the following coupled partial differential equations:

$$
\begin{aligned}
& \rho S \frac{\partial^{2} w_{1}}{\partial t^{2}}+E I \frac{\partial^{4} w_{1}}{\partial \xi^{4}}=\sum_{j=1}^{m} M_{j} \frac{\mathrm{d}}{\mathrm{d} \xi} \delta_{\xi_{j}}-\sum_{j=1}^{m} F_{j} \delta_{\xi_{j}}, \\
& \rho S \frac{\partial^{2} w_{2}}{\partial t^{2}}+E I \frac{\partial^{4} w_{2}}{\partial \xi^{4}}=\sum_{j=1}^{m} M_{j} \frac{\mathrm{d}}{\mathrm{d} \xi} \delta_{\xi_{j}}+\sum_{j=1}^{m} F_{j} \delta_{\xi_{j}},
\end{aligned}
$$

where $M_{j}$ and $F_{j}$ are given by formulas (2) and (3) in which $\varphi_{j}=(1 / 2)\left(\left.\left(\partial w_{1} / \partial \xi\right)\right|_{\xi=\xi_{j}}+\left.\left(\partial w_{2} / \partial \xi\right)\right|_{\xi=\xi_{j}}\right), \dot{\varphi}$ is the time derivative of the latter expression, $\delta_{\xi_{j}}$ is Dirac's delta, and $(\mathrm{d} / \mathrm{d} \xi) \delta_{\xi_{j}}$ is the unit doublet at $\xi=\xi_{j}$.

We build FE model of the system to design the control strategy. The original computer code for numerical simulation was elaborated for this purpose. The characteristic matrices describing the finite element of length $s$ of discretized continuous system (5) were developed. In order to obtain the virtual work of the system, we multiply the motion equations of Beam 1 and Beam 2 by virtual displacements $w_{1}^{*}(\xi)$ and $w_{2}^{*}(\xi)$, respectively. After rearrangements, the total virtual energy in the element containing both beams is equal to the integrals as follows:

$$
\begin{aligned}
& \int_{0}^{s} w_{1}^{*}(\xi)\left\{\rho S \ddot{w}_{1}+E I w_{1}^{\prime \prime \prime \prime}+h_{1} \sum_{j=1}^{m}\left[a b h\left(w_{1}^{\prime}\left(\xi_{j}\right)+w_{2}^{\prime}\left(\xi_{j}\right)\right) \delta_{\xi_{j}}^{\prime}+\frac{b h}{a}\left(w_{1}\left(\xi_{j}\right)-w_{2}\left(\xi_{j}\right)\right) \delta_{\xi_{j}}\right]\right. \\
& \left.+h_{2} \sum_{j=1}^{m}\left[a b h\left(\dot{w}_{1}^{\prime}\left(\xi_{j}\right)+\dot{w}_{2}^{\prime}\left(\xi_{j}\right)\right) \delta_{\xi_{j}}^{\prime}+\frac{b h}{a}\left(\dot{w}_{1}\left(\xi_{j}\right)-\dot{w}_{2}\left(\xi_{j}\right)\right) \delta_{\xi_{j}}\right]\right\} \mathrm{d} \xi=0, \\
& \int_{0}^{s} w_{2}^{*}(\xi)\left\{\rho S \ddot{w}_{2}+E I w_{2}^{\prime \prime \prime \prime}+h_{1} \sum_{j=1}^{m}\left[a b h\left(w_{1}^{\prime}\left(\xi_{j}\right)+w_{2}^{\prime}\left(\xi_{j}\right)\right) \delta_{\xi_{j}}^{\prime}+\frac{b h}{a}\left(w_{1}\left(\xi_{j}\right)-w_{2}\left(\xi_{j}\right)\right) \delta_{\xi_{j}}\right]\right. \\
& \left.+h_{2} \sum_{j=1}^{m}\left[a b h\left(\dot{w}_{1}^{\prime}\left(\xi_{j}\right)+\dot{w}_{2}^{\prime}\left(\xi_{j}\right)\right) \delta_{\xi_{j}}^{\prime}+\frac{b h}{a}\left(\dot{w}_{1}\left(\xi_{j}\right)-\dot{w}_{2}\left(\xi_{j}\right)\right) \delta_{\xi_{j}}\right]\right\} \mathrm{d} \xi=0,
\end{aligned}
$$


where the prime and the dot denote differentiation with respect to spatial variable $\xi$ and time variable $t$. As a result of the integration of the terms with distributions, (6) can be written in the following form:

$$
\begin{aligned}
& \int_{0}^{s} w_{1}^{*}(\xi)\left(\rho S \ddot{w}_{1}+E I w_{1}^{\prime \prime \prime \prime}\right) d \xi \\
& -h_{1} \sum_{j=1}^{m}\left[a b h w_{1}^{* \prime}\left(\xi_{j}\right)\left(w_{1}^{\prime}\left(\xi_{j}\right)+w_{2}^{\prime}\left(\xi_{j}\right)\right)\right. \\
& \left.+\frac{b h}{a} w_{1}^{*}\left(\xi_{j}\right)\left(w_{1}\left(\xi_{j}\right)-w_{2}\left(\xi_{j}\right)\right)\right] \\
& -h_{2} \sum_{j=1}^{m}\left[a b h w_{1}^{* \prime}\left(\xi_{j}\right)\left(\dot{w}_{1}^{\prime}\left(\xi_{j}\right)+\dot{w}_{2}^{\prime}\left(\xi_{j}\right)\right)\right. \\
& \left.+\frac{b h}{a} w_{1}^{*}\left(\xi_{j}\right)\left(\dot{w}_{1}\left(\xi_{j}\right)-\dot{w}_{2}\left(\xi_{j}\right)\right)\right]=0, \\
& \int_{0}^{s} w_{2}^{*}(\xi)\left(\rho S \ddot{w}_{2}+E I w_{2}^{\prime \prime \prime \prime}\right) d \xi \\
& -h_{1} \sum_{j=1}^{m}\left[a b h w_{2}^{* \prime}\left(\xi_{j}\right)\left(w_{1}^{\prime}\left(\xi_{j}\right)+w_{2}^{\prime}\left(\xi_{j}\right)\right)\right. \\
& \left.-\frac{b h}{a} w_{2}^{*}\left(\xi_{j}\right)\left(w_{1}\left(\xi_{j}\right)-w_{2}\left(\xi_{j}\right)\right)\right] \\
& -h_{2} \sum_{j=1}^{m}\left[a b h w_{2}^{* \prime}\left(\xi_{j}\right)\left(\dot{w}_{1}^{\prime}\left(\xi_{j}\right)+\dot{w}_{2}^{\prime}\left(\xi_{j}\right)\right)\right. \\
& \left.-\frac{b h}{a} w_{2}^{*}\left(\xi_{j}\right)\left(\dot{w}_{1}\left(\xi_{j}\right)-\dot{w}_{2}\left(\xi_{j}\right)\right)\right]=0 .
\end{aligned}
$$

In the case of the Euler beam, the nodal displacements are associated with the rotation angles. Hence, the displacements approximation for both beams in the finite element are given by

$$
\begin{aligned}
& w_{1}(\xi, t)=N_{1} w_{\mathrm{L} 1}+N_{2} \varphi_{\mathrm{L} 1}+N_{3} w_{\mathrm{R} 1}+N_{4} \varphi_{\mathrm{R} 1}, \\
& w_{2}(\xi, t)=N_{1} w_{\mathrm{L} 2}+N_{2} \varphi_{\mathrm{L} 2}+N_{3} w_{\mathrm{R} 2}+N_{4} \varphi_{\mathrm{R} 2} .
\end{aligned}
$$

Subscripts $L$ and $R$ denote left and right node of the finite element, respectively. The nodal displacements and angles in the element are interpolated by the 3rd-order polynomials as follows:

$$
\begin{aligned}
& N_{1}=1-3 \frac{\xi^{2}}{s^{2}}+2 \frac{\xi^{3}}{s^{3}}, \\
& N_{2}=\xi-2 \frac{\xi^{2}}{s}+\frac{\xi^{3}}{s^{2}}, \\
& N_{3}=3 \frac{\xi^{2}}{s^{2}}-2 \frac{\xi^{3}}{s^{3}}, \\
& N_{4}=-\frac{\xi^{2}}{s}+\frac{\xi^{3}}{s^{2}} .
\end{aligned}
$$

More details can be found in [18]; for example, taking into account the virtual hat-shaped function in the form

$$
\begin{aligned}
& w_{1}^{*}(\xi, t)=N_{1} w_{\mathrm{L} 1}+N_{2} \varphi_{\mathrm{L} 1}+N_{3} w_{\mathrm{R} 1}+N_{4} \varphi_{\mathrm{R} 1}, \\
& w_{2}^{*}(\xi, t)=N_{1} w_{\mathrm{L} 2}+N_{2} \varphi_{\mathrm{L} 2}+N_{3} w_{\mathrm{R} 2}+N_{4} \varphi_{\mathrm{R} 2},
\end{aligned}
$$

we can obtain the characteristic matrices of the considered system. The classical energy minimization leads to the following local inertia matrix $\mathbf{M}_{e}$ and the local stiffness matrix $\mathbf{K}_{e}$ of both beams:

$$
\mathbf{M}_{e}=\rho S\left[\begin{array}{cccccccc}
\frac{13 s}{35} & \frac{11 s^{2}}{210} & \frac{9 s}{70} & -\frac{13 s^{2}}{420} & 0 & 0 & 0 & 0 \\
\frac{11 s^{2}}{210} & \frac{s^{3}}{105} & \frac{13 s^{2}}{420} & -\frac{s^{3}}{140} & 0 & 0 & 0 & 0 \\
\frac{9 s}{70} & \frac{13 s^{2}}{420} & \frac{13 s}{35} & -\frac{11 s^{2}}{210} & 0 & 0 & 0 & 0 \\
-\frac{13 s^{2}}{420} & -\frac{s^{3}}{140} & -\frac{11 s^{2}}{210} & \frac{s^{3}}{105} & 0 & 0 & 0 & 0 \\
0 & 0 & 0 & 0 & \frac{13 s}{35} & \frac{11 s^{2}}{210} & \frac{9 s}{70} & -\frac{13 s^{2}}{420} \\
0 & 0 & 0 & 0 & \frac{11 s^{2}}{210} & \frac{s^{3}}{105} & \frac{13 s^{2}}{420} & -\frac{s^{3}}{140} \\
0 & 0 & 0 & 0 & \frac{9 s}{70} & \frac{13 s^{2}}{420} & \frac{13 s}{35} & -\frac{11 s^{2}}{210} \\
0 & 0 & 0 & 0 & -\frac{13 s^{2}}{420} & -\frac{s^{3}}{140} & -\frac{11 s^{2}}{210} & \frac{s^{3}}{105}
\end{array}\right]
$$




$$
\mathbf{K}_{e}=E I\left[\begin{array}{cccccccc}
\frac{12}{s^{3}} & \frac{6}{s^{2}} & -\frac{12}{s^{3}} & \frac{6}{s^{2}} & 0 & 0 & 0 & 0 \\
\frac{6}{s^{2}} & \frac{4}{s} & -\frac{6}{s^{2}} & \frac{2}{s} & 0 & 0 & 0 & 0 \\
-\frac{12}{s^{3}} & -\frac{6}{s^{2}} & \frac{12}{s^{3}} & -\frac{6}{s^{2}} & 0 & 0 & 0 & 0 \\
\frac{6}{s^{2}} & \frac{2}{s} & -\frac{6}{s^{2}} & \frac{4}{s} & 0 & 0 & 0 & 0 \\
0 & 0 & 0 & 0 & \frac{12}{s^{3}} & \frac{6}{s^{2}} & -\frac{12}{s^{3}} & \frac{6}{s^{2}} \\
0 & 0 & 0 & 0 & \frac{6}{s^{2}} & \frac{4}{s} & -\frac{6}{s^{2}} & \frac{2}{s} \\
0 & 0 & 0 & 0 & -\frac{12}{s^{3}} & -\frac{6}{s^{2}} & \frac{12}{s^{3}} & -\frac{6}{s^{2}} \\
0 & 0 & 0 & 0 & \frac{6}{s^{2}} & \frac{2}{s} & -\frac{6}{s^{2}} & \frac{4}{s}
\end{array}\right] .
$$

Coupling matrix $\mathbf{Y}^{j}$ is presented below:

$$
\mathbf{Y}^{j}=\left[\begin{array}{cccccccc}
y_{11}^{j} & y_{12}^{j} & y_{13}^{j} & y_{14}^{j} & \bar{y}_{11}^{j} & \bar{y}_{12}^{j} & \bar{y}_{13}^{j} & \bar{y}_{14}^{j} \\
y_{21}^{j} & y_{22}^{j} & y_{23}^{j} & y_{24}^{j} & \bar{y}_{21}^{j} & \bar{y}_{22}^{j} & \bar{y}_{23}^{j} & \bar{y}_{24}^{j} \\
y_{31}^{j} & y_{32}^{j} & y_{33}^{j} & y_{34}^{j} & \bar{y}_{31}^{j} & \bar{y}_{32}^{j} & \bar{y}_{33}^{j} & \bar{y}_{34}^{j} \\
y_{41}^{j} & y_{42}^{j} & y_{43}^{j} & y_{44}^{j} & \bar{y}_{41}^{j} & \bar{y}_{42}^{j} & \bar{y}_{43}^{j} & \bar{y}_{44}^{j} \\
\bar{y}_{11}^{j} & \bar{y}_{12}^{j} & \bar{y}_{13}^{j} & \bar{y}_{14}^{j} & y_{11}^{j} & y_{12}^{j} & y_{13}^{j} & y_{14}^{j} \\
\bar{y}_{21}^{j} & \bar{y}_{22}^{j} & \bar{y}_{23}^{j} & \bar{y}_{24}^{j} & y_{21}^{j} & y_{22}^{j} & y_{23}^{j} & y_{24}^{j} \\
\bar{y}_{31}^{j} & \bar{y}_{32}^{j} & \bar{y}_{33}^{j} & \bar{y}_{34}^{j} & y_{31}^{j} & y_{32}^{j} & y_{33}^{j} & y_{34}^{j} \\
\bar{y}_{41}^{j} & \bar{y}_{42}^{j} & \bar{y}_{43}^{j} & \bar{y}_{44}^{j} & y_{41}^{j} & y_{42}^{j} & y_{43}^{j} & y_{44}^{j}
\end{array}\right],
$$

where, according to the shape functions (9),

$$
\begin{aligned}
& y_{p q}^{j}=-a b h N_{p}^{\prime}\left(\xi_{j}\right) N_{q}^{\prime}\left(\xi_{j}\right)-\frac{b h}{a} N_{p}\left(\xi_{j}\right) N_{q}\left(\xi_{j}\right), \\
& \bar{y}_{p q}^{j}=-a b h N_{p}^{\prime}\left(\xi_{j}\right) N_{q}^{\prime}\left(\xi_{j}\right)+\frac{b h}{a} N_{p}\left(\xi_{j}\right) N_{q}\left(\xi_{j}\right) .
\end{aligned}
$$

Let $n$ be the number of nodes of each beam and $\mathbf{T}=$ $\left[T_{1}, \ldots, T_{4 n}\right]^{\mathrm{T}}$ be the vector of displacements and angles of the nodes of both beams. Finally, according to (7), the dynamics are governed by the following second-order differential equation:

$$
\mathbf{M} \ddot{\mathbf{T}}+\mathbf{K T}+\sum_{j=1}^{m} h_{2}\left(u_{j}\right) \mathbf{Y}^{j} \dot{\mathbf{T}}+\sum_{j=1}^{m} h_{1}\left(u_{j}\right) \mathbf{Y}^{j} \mathbf{T}=\mathbf{0},
$$

where $\mathbf{M}$ is the global mass matrix, $\mathbf{K}$ is global stiffness matrix, and $\mathbf{Y}$ is matrix that establishes the effect of the damping block. Global matrices $\mathbf{M}$ and $\mathbf{K}$ are assembled from elemental matrices $\mathbf{M}_{e}$ and $\mathbf{K}_{e}$. Matrices $\mathbf{Y}^{j}$ are assembled in matrix places related to positions of the coupling blocks.

\section{Control Design}

For the sake of the further study, we will now introduce the state-space first-order ordinary differential representation of the dynamics (14). For state vector $\mathbf{x} \in \mathbb{R}^{8 n}$ defined as

$$
\begin{aligned}
\mathbf{x} & =\left[\mathbf{x}_{\mathrm{I}}, \mathbf{x}_{\mathrm{II}}\right]^{\mathrm{T}}, \\
\mathbf{x}_{\mathrm{I}} & =\left[T_{1}, T_{2}, \ldots, T_{4 n}\right], \\
\mathbf{x}_{\mathrm{II}} & =\left[\dot{T}_{1}, \dot{T}_{2}, \ldots, \dot{T}_{4 n}\right],
\end{aligned}
$$

we introduce system matrices $\mathbf{A}_{(8 n \times 8 n)}, \mathbf{B}_{1(8 n \times 8 n)}^{j}, \mathbf{B}_{2(8 n \times 8 n)}^{j}$ given by

$$
\begin{aligned}
\mathbf{A} & =\left[\begin{array}{cc}
\mathbf{0} & \mathbf{I} \\
-\mathbf{M}^{-1} \mathbf{K} & \mathbf{0}
\end{array}\right], \\
\mathbf{B}_{1}^{j} & =\left[\begin{array}{cc}
\mathbf{0} & \mathbf{0} \\
-\mathbf{M}^{-1} \mathbf{Y}^{j} & \mathbf{0}
\end{array}\right], \\
\mathbf{B}_{2}^{j} & =\left[\begin{array}{cc}
\mathbf{0} & \mathbf{0} \\
\mathbf{0} & -\mathbf{M}^{-1} \mathbf{Y}^{j}
\end{array}\right] .
\end{aligned}
$$

Then, the system dynamics given by (14) can be represented by the following system of the ordinary differential equations:

$$
\begin{aligned}
\dot{\mathbf{x}}(t)= & \mathbf{A x}(t)+\sum_{j=1}^{m} h_{1}\left(u_{j}\right) \mathbf{B}_{1}^{j} \mathbf{x}(t) \\
& +\sum_{j=1}^{m} h_{2}\left(u_{j}\right) \mathbf{B}_{2}^{j} \mathbf{x}(t), \\
\mathbf{x}(0)= & \mathbf{x}_{\mathbf{0}} .
\end{aligned}
$$


Here, $\mathbf{x}_{\mathbf{0}}$ stands for a nonzero initial condition. For the control inputs, we assume that each is bounded by two nonnegative values corresponding to the minimum and maximum admissible magnitude of the magnetic field denoted by $u_{\min }$ and $u_{\text {max }}$, respectively; namely,

$$
u_{j} \in \mathscr{U}=\left[u_{\min }, u_{\max }\right] \subset \mathbb{R}_{\geq 0}, \quad j=1,2, \ldots, m .
$$

Note that control variable $u$ appears linearly in control system (17). Moreover, due to the middle term in the right-hand side of (17), the system is classified as a bilinear [19]. By PicardLindelöf theorem for given function $u$, the initial boundary value problem (17) possesses unique solution $\mathbf{x}(t)$. For the numerical results presented in the sequel of this work, the dynamics (17) will be solved by employing the 4 th-order Runge-Kutta scheme.

Before we design a controller, let us first recall the result from $[1,20]$. It has been demonstrated that the optimal policy to stabilize the bilinear systems as (17) is realized by the socalled bang-bang control laws, where the control parameter is switched between two extreme values of the admissible set. The result is also valid for optimal control of any bilinear system with a finite time integral objective functional that does not explicitly depend on the control parameter, as in the cases of energy-related functionals. Therefore, unless there are strong practical reasons to reduce the amount of control input, the usage of controls other than bang-bang controls has no rational argument. To determine an exact structure of the optimal bang-bang control, one needs to estimate the number of switches and the corresponding time instants (referred to as the switching times). Practically, this requires solving a two-point boundary value problem that, for the multidimensional system, is concerned with employment of significant amount of computational burden. Another inconvenience of the optimal bang-bang approach is due to the fact that the structures of the optimal controls strictly depend on the system's initial conditions. Since we are aiming at a controller that is robust for any initial excitement, we will opt for an alternative method.

In the present work, a switched control strategy will be designed based on Lyapunov's classical second method for stability. It will enable us to derive an explicit, simple, and robust state-feedback control law capable of real-time implementation with the use of accessible state information. For this purpose, let us first introduce the energy-related Lyapunov function corresponding to system (17):

$$
V(\mathbf{x}(t))=\frac{1}{2} \mathbf{x}(t)^{\mathrm{T}} \mathbf{Q} \mathbf{x}(t), \quad \mathbf{Q}>0, \mathbf{Q}=\mathbf{Q}^{\mathrm{T}}
$$

The time derivative of the energy function is

$$
\dot{V}(\mathbf{x}(t))=\frac{1}{2} \dot{\mathbf{x}}^{\mathrm{T}} \mathbf{Q} \mathbf{x}+\frac{1}{2} \mathbf{x}^{\mathrm{T}} \mathbf{Q} \dot{\mathbf{x}} .
$$

Our goal is to design controls $u_{j}^{*}$ providing the best instantaneous energy dissipation; namely,

$$
\forall t: u_{j}^{*}(t)=\underset{u_{j} \in \mathcal{U}}{\arg \min } \dot{V}(\mathbf{x}(t)), \quad j=1,2, \ldots, m .
$$

To find a solution to problem (21), we first insert dynamical equation (17) into (20). That yields

$$
\begin{aligned}
\dot{V}= & \mathbf{x}^{\mathrm{T}} \mathbf{A}^{\mathrm{T}} \mathbf{Q} \mathbf{x}+\sum_{j=1}^{m} h_{1}\left(u_{j}\right) \mathbf{x}^{\mathrm{T}} \mathbf{B}_{1}^{j^{\mathrm{T}}} \mathbf{Q} \mathbf{x} \\
& +\sum_{j=1}^{m} h_{2}\left(u_{j}\right) \mathbf{x}^{\mathrm{T}} \mathbf{B}_{2}^{j^{\mathrm{T}}} \mathbf{Q} \mathbf{x} .
\end{aligned}
$$

For simplicity, let us define the following state-dependent functions:

$$
\begin{aligned}
& G_{1}^{j}(\mathbf{x})=\mathbf{x}^{\mathrm{T}} \mathbf{B}_{1}^{j^{\mathrm{T}}} \mathbf{Q} \mathbf{x}, \\
& G_{2}^{j}(\mathbf{x})=\mathbf{x}^{\mathrm{T}} \mathbf{B}_{2}^{j^{\mathrm{T}}} \mathbf{Q} \mathbf{x}, \\
& \quad j=1,2, \ldots, m .
\end{aligned}
$$

For such a representation, the solution to problem (21) can be determined by solving the following mathematical programming problems:

$$
\begin{array}{r}
u_{j}^{*}(t) \\
=\underset{u_{j} \in \mathcal{U}}{\arg \min }\left\{G_{1}^{j}(\mathbf{x}(t)) h_{1}\left(u_{j}\right)+G_{2}^{j}(\mathbf{x}(t)) h_{2}\left(u_{j}\right)\right\}, \\
j=1,2, \ldots, m .
\end{array}
$$

Assuming that, for all time $t$, state $\mathbf{x}(t)$ is accessible, we will now consider three cases depending on the values of functions $G_{1}^{j}(\mathbf{x}(t))$ and $G_{2}^{j}(\mathbf{x}(t))$.

Case $1\left(G_{1}^{j}(\mathbf{x}(t))>0\right.$ and $\left.G_{2}^{j}(\mathbf{x}(t))>0\right)$. Recall the fact that both $h_{1}\left(u_{j}\right)$ and $h_{2}\left(u_{j}\right)$ are monotonically increasing functions. Therefore, in this case, we have

$$
\begin{aligned}
& u_{j}^{*}(t) \\
& =\underset{u_{j} \in \mathcal{U}}{\arg \min }\left\{G_{1}^{j}(\mathbf{x}(t)) h_{1}\left(u_{j}\right)+G_{2}^{j}(\mathbf{x}(t)) h_{2}\left(u_{j}\right)\right\} \\
& =\underset{u_{j} \in \mathcal{U}}{\arg \min }\left\{u_{j}\right\}=u_{\min } .
\end{aligned}
$$

Case $2\left(G_{1}^{j}(\mathbf{x}(t))<0\right.$ and $\left.G_{2}^{j}(\mathbf{x}(t))<0\right)$. By analogy with Case 1 , now we have

$$
u_{j}^{*}(t)=\underset{u_{j} \in \mathscr{U}}{\arg \max }\left\{u_{j}\right\}=u_{\max } .
$$

Case $3\left(G_{1}^{j}(\mathbf{x}(t)) G_{2}^{j}(\mathbf{x}(t))<0\right)$. In this case, we need to establish whether term $G_{1}^{j}(\mathbf{x}(t)) h_{1}\left(u_{j}\right)+G_{2}^{j}(\mathbf{x}(t)) h_{2}\left(u_{j}\right)$ describes an increasing or a decreasing function of $u_{j}$. To do so, one can verify the following conditions:

$$
\begin{array}{ll}
\text { (a) } \quad & G_{1}^{j}(\mathbf{x}(t)) h_{1}\left(u_{\max }\right)+G_{2}^{j}(\mathbf{x}(t)) h_{2}\left(u_{\max }\right) \\
& >G_{1}^{j}(\mathbf{x}(t)) h_{1}\left(u_{\min }\right)+G_{2}^{j}(\mathbf{x}(t)) h_{2}\left(u_{\min }\right), \\
\text { (b) } G_{1}^{j}(\mathbf{x}(t)) h_{1}\left(u_{\max }\right)+G_{2}^{j}(\mathbf{x}(t)) h_{2}\left(u_{\max }\right) \\
\quad<G_{1}^{j}(\mathbf{x}(t)) h_{1}\left(u_{\min }\right)+G_{2}^{j}(\mathbf{x}(t)) h_{2}\left(u_{\min }\right) .
\end{array}
$$




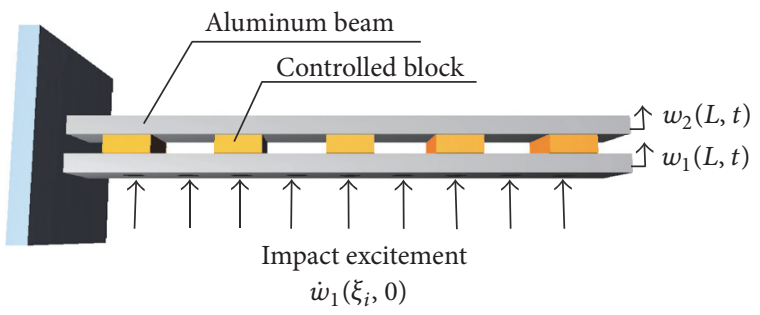

FIGURE 3: The assumed setup for the structure used in the simulations. Two aluminum beams are coupled with five controlled damping blocks. The initial conditions are imposed to the lower beam.

If (a) holds, then we have

$$
\begin{aligned}
& u_{j}^{*}(t) \\
& \quad=\underset{u_{j} \in \mathcal{U}}{\arg \min }\left\{G_{1}^{j}(\mathbf{x}(t)) h_{1}\left(u_{j}\right)+G_{2}^{j}(\mathbf{x}(t)) h_{2}\left(u_{j}\right)\right\} \\
& \quad=\underset{u_{j} \in \mathcal{U}}{\arg \min }\left\{u_{j}\right\}=u_{\text {min }} .
\end{aligned}
$$

Similarly, if (b) is fulfilled, then

$$
\begin{aligned}
& u_{j}^{*}(t) \\
& =\underset{u_{j} \in \mathcal{U}}{\arg \min }\left\{G_{1}^{j}(\mathbf{x}(t)) h_{1}\left(u_{j}\right)+G_{2}^{j}(\mathbf{x}(t)) h_{2}\left(u_{j}\right)\right\} \\
& \quad=\underset{u_{j} \in \mathcal{U}}{\arg \max }\left\{u_{j}\right\}=u_{\max } .
\end{aligned}
$$

Remarks. For the specific case where $G_{1}^{j}(\mathbf{x}(t))=0$, we assume that $u_{j}^{*}(t)=u_{\min }$ if $G_{2}^{j}(\mathbf{x}(t))>0$, and $u_{j}^{*}(t)=u_{\max }$ if $G_{2}^{j}(\mathbf{x}(t))<0$. Similarly, for the case where $G_{2}^{j}(\mathbf{x}(t))=0$, we assume that $u_{j}^{*}(t)=u_{\min }$ if $G_{1}^{j}(\mathbf{x}(t))>0$, and $u_{j}^{*}(t)=u_{\max }$ if $G_{1}^{j}(\mathbf{x}(t))<0$. Finally, in the singular case when $G_{1}^{j}(\mathbf{x}(t))=0$ and $G_{2}^{j}(\mathbf{x}(t))=0$, we can apply any control value $u_{j}^{*}(t) \in \mathscr{U}$.

The proposed switching control can be implemented with commonly accessible instrumentation. To reconstruct the state vector, one can use image data acquisition system. The computation burden used for Cases $1-3$ is minimal. To verify the actual condition, one needs to resolve simple algebraic calculation. As a result, the solution to problem (24) can be attained rapidly, even with the use of a standard PC. To avoid the chattering effect concerned with the switched controls, in the practical realization, one may consider applying a smooth approximation of the switching control (see, e.g., [21]).

\section{Numerical Study}

In this section, we will perform a set of numerical experiments to validate the designed controller. Our primary goal will be to examine its robustness when the structure is subjected to different types of the impact excitations. For that purpose, the simulations will be performed for different initial condition scenarios.
For the considered structure, we assume two identical aluminum beams coupled by five identical controlled damping blocks (see Figure 3). For a such system, we select a set of parameters listed as follows.

\section{Model Parameters Used in the Simulations}

\section{Beam Parameters}

Beam length $(L): 1[\mathrm{~m}]$

Young modulus $(E)$ : $69[\mathrm{GPa}]$

Moment of inertia $(I): 7.03 \times 10^{-12}\left[\mathrm{~m}^{4}\right]$

Density per length $(\rho): 0.101[\mathrm{~kg} / \mathrm{m}]$

\section{Damping Block Parameters}

$$
\begin{aligned}
& \text { Half of block length }(a): 0.0175[\mathrm{~m}] \\
& \text { Block width }(b): 0.0250[\mathrm{~m}] \\
& \text { Half of block depth }(h): 0.005[\mathrm{~m}] \\
& \text { Storage modulus at } u=0\left(G_{0}^{\prime}\right): 5 \times 10^{4}[\mathrm{~Pa}] \\
& \text { Loss modulus at } u=0\left(G_{0}^{\prime \prime}\right): 9 \times 10^{3}[\mathrm{~Pa}] \\
& \text { Approximated first natural frequency }(\omega): 10[\mathrm{~Hz}] \\
& \text { Relative MRE effect for } G^{\prime}\left(r_{1}\right): 1.4[\cdot] \\
& \text { Relative MRE effect for } G^{\prime \prime}\left(r_{2}\right): 1.5[\cdot]
\end{aligned}
$$

In the simulations, we will use the finite element model introduced in Section 2, where, for each of the beams, we will assume ten equal elements placed among eleven nodes. The controlled blocks are located at positions $(1 / 10) L$, $(3 / 10) L,(5 / 10) L,(7 / 10) L$, and $(9 / 10) L$. The simulations will be performed for the time horizon of $T=3 \mathrm{~s}$. To establish the comparative results, we will utilize the total energy metric defined as follows:

$$
J=\frac{1}{2} \int_{0}^{T}\left\{\mathbf{x}_{\mathrm{I}}^{T}(t) \mathbf{K} \mathbf{x}_{\mathrm{I}}(t)+\mathbf{x}_{\mathrm{II}}^{T}(t) \mathbf{M} \mathbf{x}_{\mathrm{II}}(t)\right\} \mathrm{d} t .
$$

Here, $\mathbf{K}$ and $\mathbf{M}$ are defined according to (14). For the extreme values of the admissible control (see (18)), we will assume $u_{\min }=0$ and $u_{\max }=1$. Control variable $u$ denotes the intensity of the field in the dimensionless form; that is, this is the actual value divided by the maximum one.

For the assumed finite element representation, we introduce $\mathscr{I}_{1}=\{1, \ldots, 11\}$ and $\mathscr{I}_{2}=\{12, \ldots, 22\}$ standing for the set of nodes associated with the lower and upper beam, respectively. Let $\xi_{i}$ stand for the horizontal position of $i$ th node. We will consider the following three variants of the initial condition for system (5).

Variant 1. In this case, we will be interested in how the designed control can stabilize the structure subjected to a single earth tremor or a strong wind blow. For that purpose, we will impose the initial velocity distributed over the set of 
nodes of the lower beam. For the upper beam, we will assume the zero initial condition.

$$
\begin{array}{ll}
w_{1}\left(\xi_{i}, 0\right) & =0, \quad i \in \mathscr{I}_{1}, \\
\dot{w}_{1}\left(\xi_{1}, 0\right) & =0, \\
\dot{w}_{1}\left(\xi_{i}, 0\right) & =0.001 i^{3}[\mathrm{~m} / \mathrm{s}], \quad i \in \mathscr{I}_{1} \backslash\{1\}, \\
w_{2}\left(\xi_{i}, 0\right) & =0, \\
\dot{w}_{2}\left(\xi_{i}, 0\right) & =0, \\
& i \in \mathscr{I}_{2} .
\end{array}
$$

Variant 2. In this case, we will examine the performance of the controller when the structure is subjected to concentrated impact excitement. We will impose an initial velocity to the node placed at the end of the lower beam. As before, for the upper beam, we will assume the zero initial condition.

$$
\begin{aligned}
w_{1}\left(\xi_{i}, 0\right) & =0, \quad i \in \mathscr{I}_{1}, \\
\dot{w}_{1}\left(\xi_{11}, 0\right) & =5[\mathrm{~m} / \mathrm{s}], \\
\dot{w}_{1}\left(\xi_{i}, 0\right) & =0, \quad i \in \mathscr{I}_{1} \backslash\{11\}, \\
w_{2}\left(\xi_{i}, 0\right) & =0, \\
\dot{w}_{2}\left(\xi_{i}, 0\right) & =0,
\end{aligned}
$$

$$
i \in \mathscr{I}_{2} \text {. }
$$

Variant 3. In this variant, we will consider a concentrated impact excitement subjected to the middle point of the lower beam. The zero initial condition is assumed for the upper beam.

$$
\begin{aligned}
& w_{1}\left(\xi_{i}, 0\right)=0, \quad i \in \mathscr{I}_{1}, \\
& \dot{w}_{1}\left(\xi_{6}, 0\right)=10[\mathrm{~m} / \mathrm{s}], \\
& \dot{w}_{1}\left(\xi_{i}, 0\right)=0, \quad i \in \mathscr{I}_{1} \backslash\{6\}, \\
& w_{2}\left(\xi_{i}, 0\right)=0, \\
& \dot{w}_{2}\left(\xi_{i}, 0\right)=0,
\end{aligned}
$$

$$
i \in \mathscr{I}_{2} \text {. }
$$

4.1. The Optimal Passive Cases. The first set of numerical experiments is performed to establish the optimal passive strategy to serve as a comparative result to justify the effectiveness of the designed controller. We consider five passive strategies where for each block the control parameter is set to constant value of $u=0, u=0.25, u=0.50$, $u=0.75$, and $u=1$, respectively. For the initial condition of variant 1 , the comparison of the deflection of the ending point of lower beam $w_{1}(L, t)$ is demonstrated in Figure 4 . We can clearly observe that the increase of the value of the control parameter results in higher rates of decrease of the vibration amplitude. The same result applies to the upper beam. For the assumed metric (30), over $46 \%$ benefit is achieved for

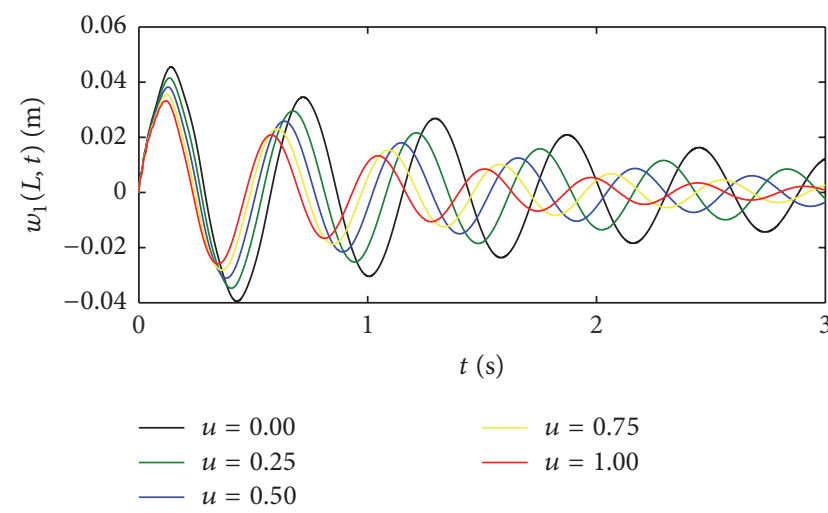

FIGURE 4: Comparison of the displacement of lower beam $w_{1}(L, t)$ for the passive cases in variant 1 .

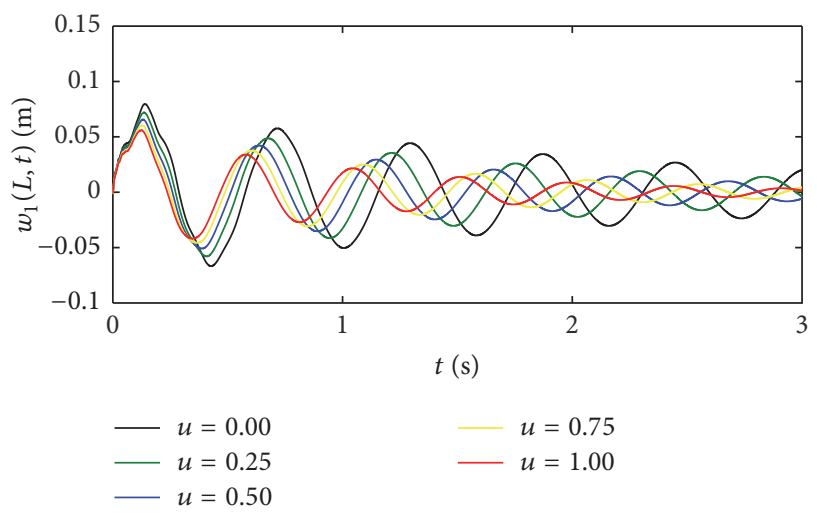

FIGURE 5: Comparison of the displacement of lower beam $w_{1}(L, t)$ for the passive cases in variant 2 .

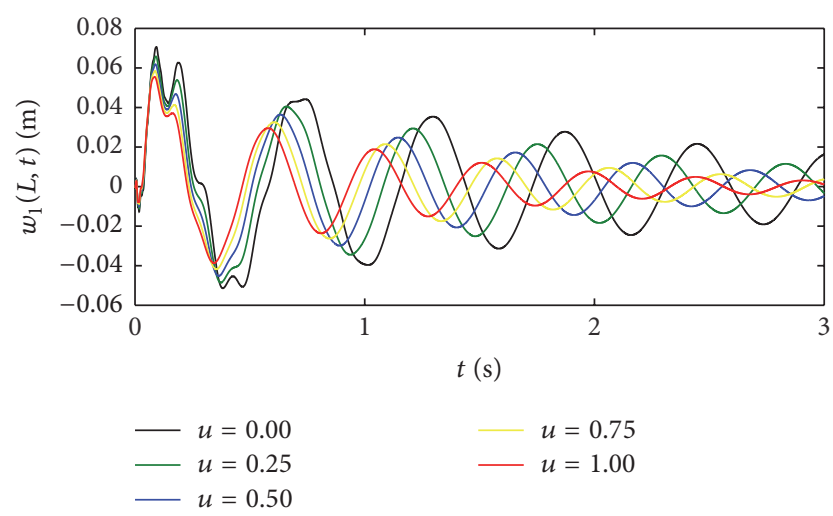

FIGURE 6: Comparison of the displacement of lower beam $w_{1}(L, t)$ for the passive cases in variant 3 .

$u=1$ compared to the case of $u=0$. A similar conclusion is observed for the initial conditions of variants 2 and 3 (Figures 5 and 6 ) where the total energy is reduced by $48 \%$ and $44 \%$, respectively (see Table 1 for the full set of the energy metric values). The presented results confirm the expected dynamical behavior since the value of the control parameter is related to the structural damping parameters (see (1)). To examine the performance of the designed controller, for each 


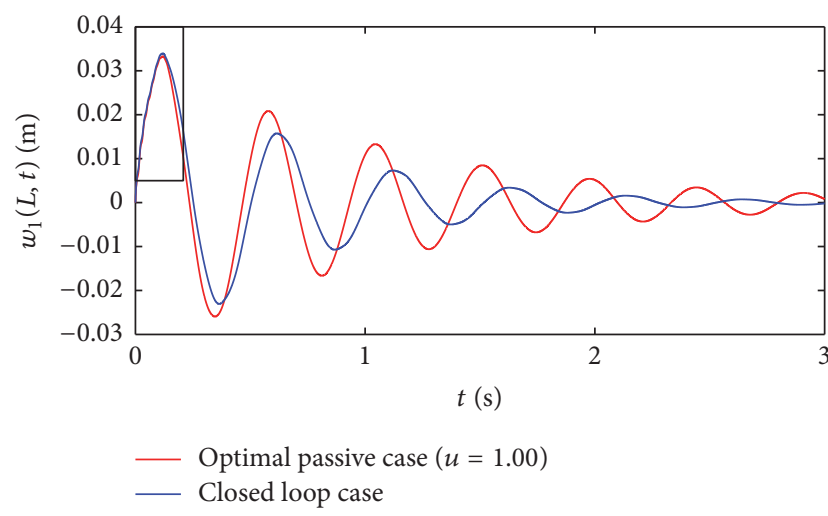

(a)

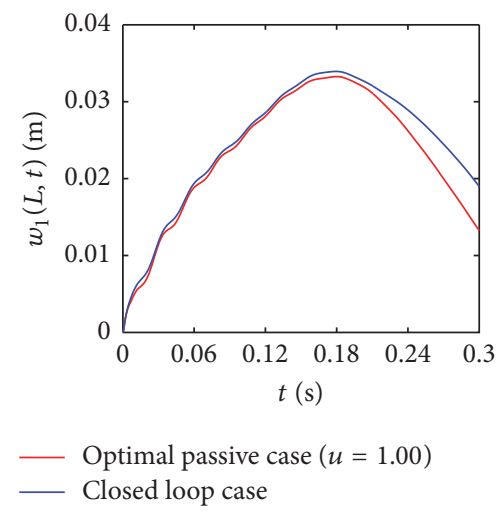

(b)

FIGURE 7: Comparison of the displacement of lower beam $w_{1}(L, t)$ for the optimal passive case and the closed loop case in variant 1 (a) and the zoomed visualisation of the primary extreme deflection (b).

TABLE 1: The comparison of total energy $J$. For each of the variants, the values are normalized to case $u=0$.

\begin{tabular}{cccccc}
\hline & $u=0.00$ & $u=0.25$ & $u=0.50$ & $u=0.75$ & $u=1.00$ \\
\hline Variant 1 & 1.000 & 0.756 & 0.606 & 0.507 & 0.436 \\
Variant 2 & 1.000 & 0.749 & 0.595 & 0.494 & 0.423 \\
Variant 3 & 1.000 & 0.757 & 0.614 & 0.521 & 0.457 \\
\hline
\end{tabular}

initial condition variant, we will use the passive strategies $u=1$, later referred to as the optimal passive cases.

4.2. Switched Controller versus the Optimal Passive Cases. For the testing of the designed controller, we begin with the simulations under the initial condition of variant 1 . The comparison of the deflection of the ending point of lower beam $w_{1}(L, t)$ for the controlled case (referred to as closed loop case) and the optimal passive case is depicted in Figure 7. At the first stage of the simulations, we observe that both trajectories are almost identical which is due to the fact that, for each of the blocks, the switching controller mainly operates on maximum admissible value $u_{\max }=1$. After reaching the first maximal deflection (at time $t \approx 0.18 \mathrm{~s}$ ), we notice a gradual divergence of the curves. After this time, we can observe a decrease of the velocity (inspected by the lower slope) in the closed loop case. This velocity decrease results in $16 \%$ reduction of the second extreme deflection. For the rest of the simulation, we clearly observe the tendency of faster stabilization for the closed loop case.

Switching of the control parameter influences both the damping and the stiffness of the blocks according to (1). As the control is applied, due to the temporary stiffness loss, the peak frequencies slightly shift towards the lower values (Figure 10). The amplitude is presented as a relative strength of the harmonic component of the source signal. For each frequency, the damping ratio was estimated using half-power bandwidth method and plotted on the frequency response graph. The damping ratio for the optimal passive case is denoted $\zeta_{p}$, while $\zeta_{c}$ is used for the controlled, closed loop case. One can notice that the controlled case outperforms
TABLE 2: The values of total energy $J$ normalized to those of optimal passive case $u=1$.

\begin{tabular}{lcc}
\hline Variant 1 & Variant 2 & Variant 3 \\
\hline 0.689 & 0.670 & 0.724 \\
\hline
\end{tabular}

the optimal passive case for each variant. Still, the damping effectiveness differs.

In Figure 11, we illustrate the switching control modes of the damping blocks at time instants $t=0.002 \mathrm{~s}, t=0.02 \mathrm{~s}$, $t=0.2 \mathrm{~s}$, and $t=2 \mathrm{~s}$. Here, for each of the blocks, red and blue colors correspond to switching modes $u_{\max }$ and $u_{\min }$, respectively. Note that the initial condition of variant 1 enforces the approximate shapes of the beams' first mode, so single frequency peak is observed. As a result, for all of the damping blocks, the switches triggered by the sign of the functions (23) take place nearly at the same time (due to the coherence in the changes of signs of the state vector terms), and thus their switching modes remain consistent. In terms of the assumed energy metric, the closed loop case outperforms the optimal passive case by over $31 \%$ (see Table 2 ). As a result of the control, the damping ratio increases from $\zeta_{p}=0.164$ to $\zeta_{c}=0.186$, that is, by $19 \%$.

The comparison of the deflection of the end point of lower beam $w_{1}(L, t)$ in the case of the initial condition of variant 2 is demonstrated in Figure 8. At the first stage of the simulation, for both trajectories, we observe a minor presence of the higher vibration modes which is due to the point impact. At this stage, the switching modes of the damping blocks are not consistent (see Figure 12). Thanks to the control policy, the first extreme deflection of the closed loop trajectory is slightly reduced. As for the previous initial condition scenario, we observe a reduction of the velocity and the second maximal deflection for the closed loop case. Afterwards, the dynamics become dominated by the first mode, and the switching pattern returns to that observed in the case of variant 1 . The overall reduction of the energy is $33 \%$ compared to the optimal passive case. When the closed loop switching control is applied, the damping ratio for the first-peak frequency 


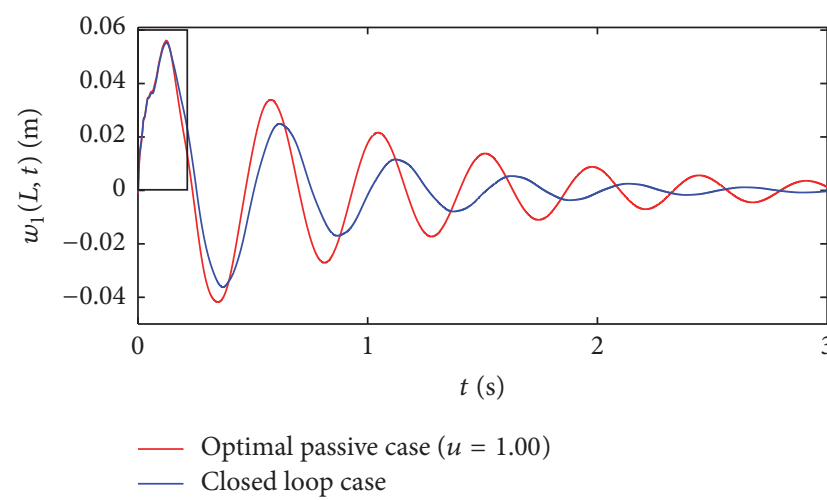

(a)

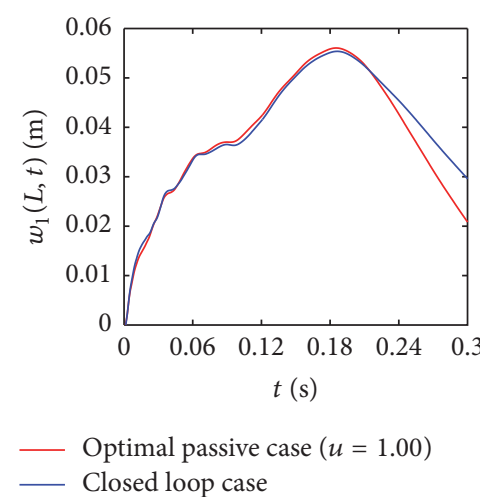

(b)

Figure 8: Comparison of the displacement of lower beam $w_{1}(L, t)$ for the optimal passive case and the closed loop case in variant 2 (a) and the zoomed visualisation of the primary extreme deflection (b).

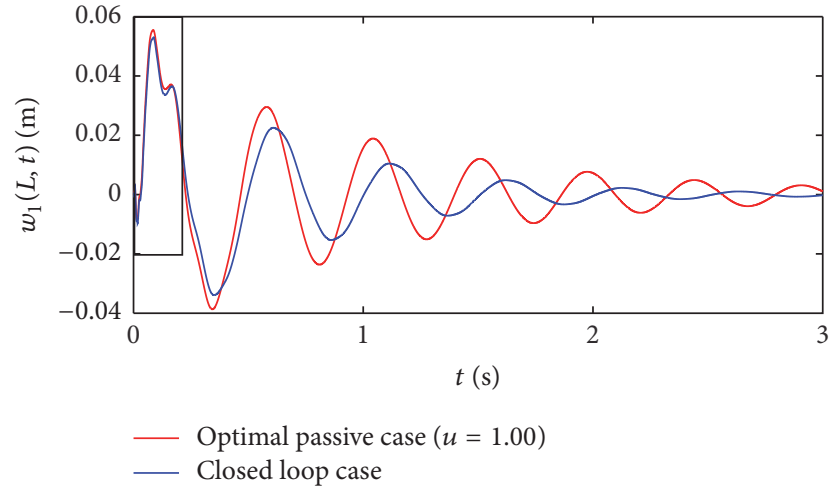

(a)

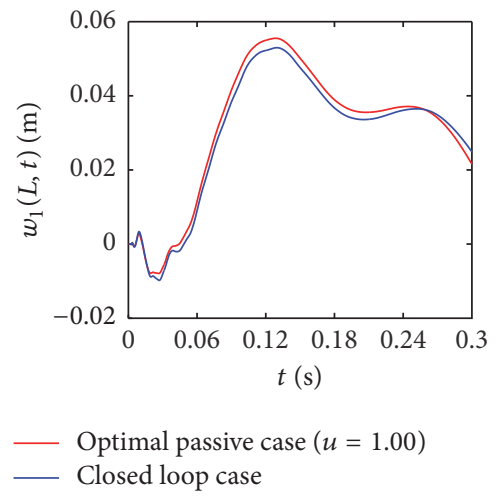

(b)

FIGURE 9: Comparison of the displacement of lower beam $w_{1}(L, t)$ for the optimal passive case and the closed loop case in variant 3 (a) and the zoomed visualisation of the primary extreme deflection (b).

increases by $22 \%$, from $\zeta_{p 1}=0.158$ up to $\zeta_{c 1}=0.193$. When the frequency of the second mode is considered, the damping increases by the same percentage, from $\zeta_{p 2}=0.133$ up to $\zeta_{c 2}=0.162$.

In Figure 9, we present the comparison of deflection $w_{1}(L, t)$ in the case of the initial condition of variant 3 . As in variant 2 , at the first stage of the simulation, we observe the presence of higher vibration modes resulting in irregular switching control patterns (see Figure 13). We can notice a minor drop of the peak amplitude for the closed loop case. After vanishing of the higher vibration modes, the dynamics and the control pattern return to those of variant 1. Compared to the optimal passive case, the closed loop system resulted in $28 \%$ reduction of the total energy metric. The passive damping ratio at the first-peak frequency $\zeta_{p 1}=0.161$ is increased by the switching control policy to $\zeta_{c 1}=0.185$, which is $15 \%$ growth. When the second mode is considered, the damping ratio for the optimal passive case is $\zeta_{p 2}=0.215$. As a result of the designed closed loop controller, this value was raised by $16 \%$ to $\zeta_{c 2}=0.250$.

Finally, we will analyze the character of damping in the system. We define the damping decrement as the ratio of consecutive extreme deflections of the lower beam (Note that multiple extremes may occur at the first cycles of vibrations of the dominating mode, which results from the fact that several natural modes are induced, and this is clearly visible in Figure 9. In such a case only the extremes of a dominating mode are taken into account.). Specifically, if $t_{i}$ denotes a time instant of $i$ th extreme deflection $\left(i=1, \ldots, N_{e}\right.$, where $N_{e}$ is the number of extremes), then the damping decrement at $t_{i}$ is equal to

$$
\delta\left(t_{i}\right)=\frac{\left|w_{1}\left(L, t_{i}\right)\right|}{\left|w_{1}\left(L, t_{i+1}\right)\right|}-1, \quad i=1, \ldots, N_{e}-1 .
$$

The values of damping decrements for optimal passive and closed loop cases are depicted in Figure 14. For every variant of initial conditions, the closed loop control almost doubles the decrease rate of the amplitude, from approximately $25 \%$ to $50 \%$. For most of the simulation, the values of decrements are constant which is a consequence of the presence of linear viscous damping. The exception is observed at the beginning of the simulation. Here, the values of the initial 2-3 decrements differ because more than one of the natural 

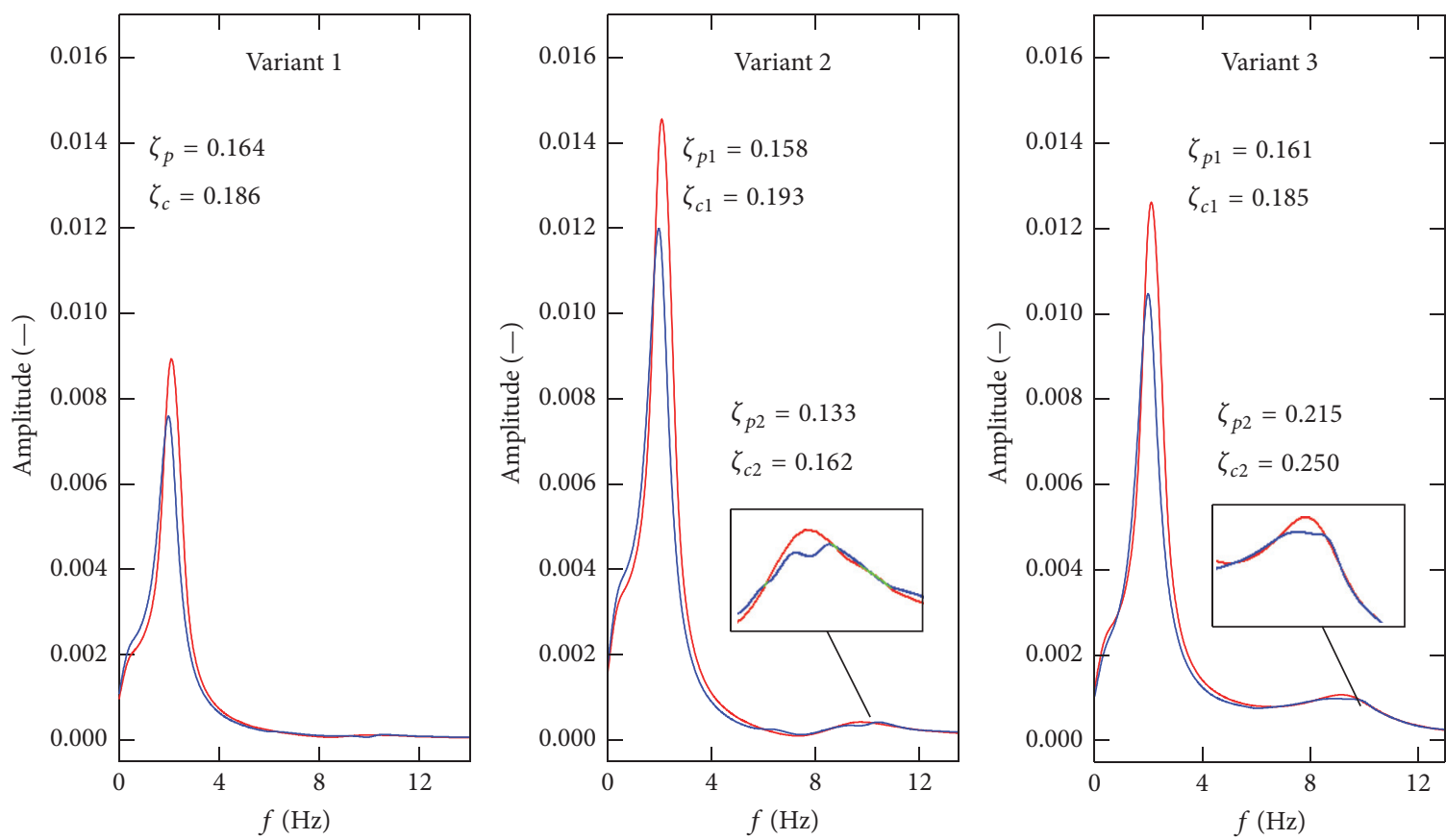

- Optimal passive case $(u=1.00)$

- Closed loop case

FIGURE 10: Comparison of the frequency and half-power bandwidth damping ratio of the lower beam for the optimal passive case and the closed loop case.
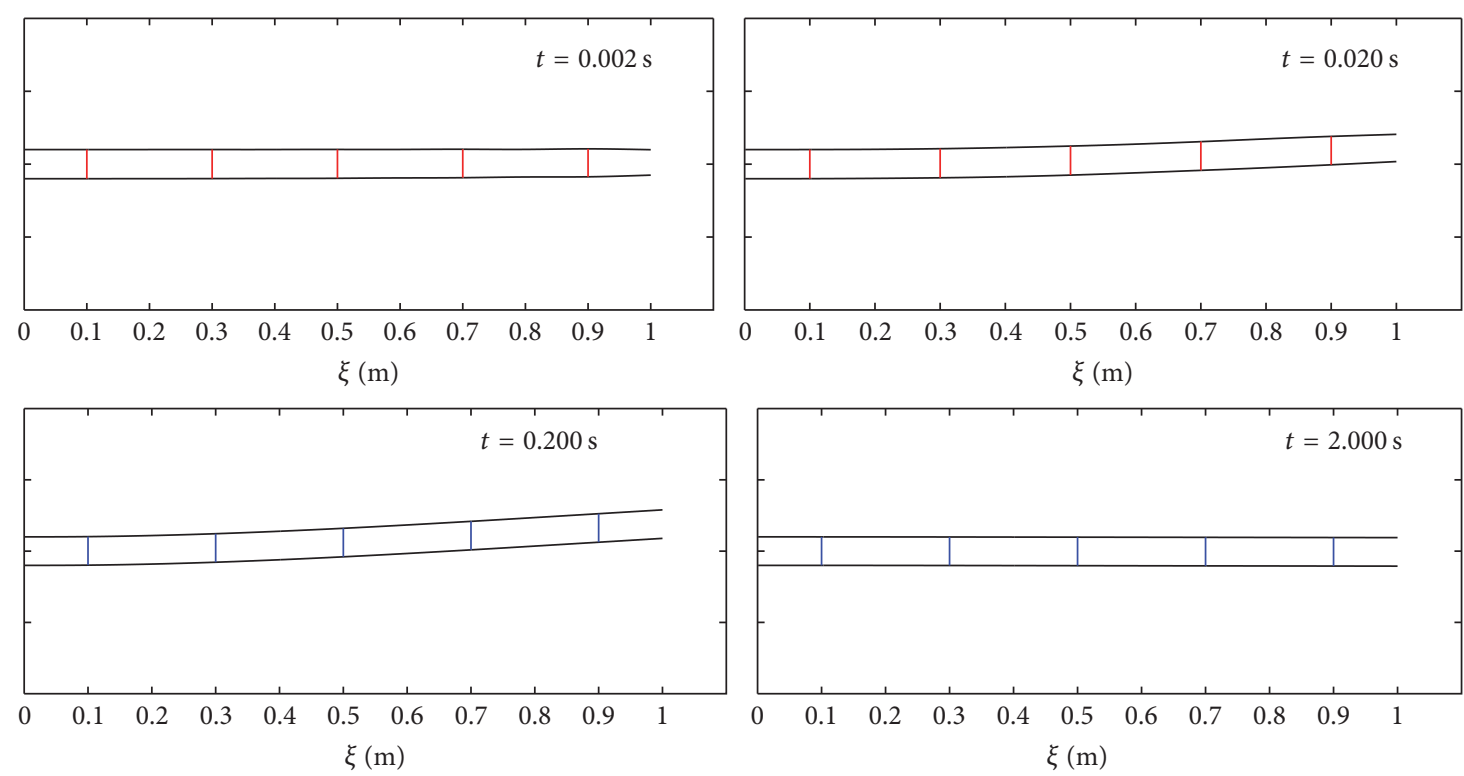

FIGURE 11: Activity of the controlled blocks for the selected time instants in the case of variant 1 . Blue and red colors correspond to $u_{j}=u_{\max }$ and $u_{j}=u_{\text {min }}$, respectively.

modes are induced. It is particularly observable in variants 2 and 3 , when the initial conditions induce several modes at once, whereas in variant 1 the initial condition given in a third-order polynomial form is closely related to the first eigenfunction of a cantilever beam.

\section{Conclusions}

In this paper, a control method to stabilize adjacent structures by means of incorporated controllable damping blocks has been designed and numerically evaluated. The controller 

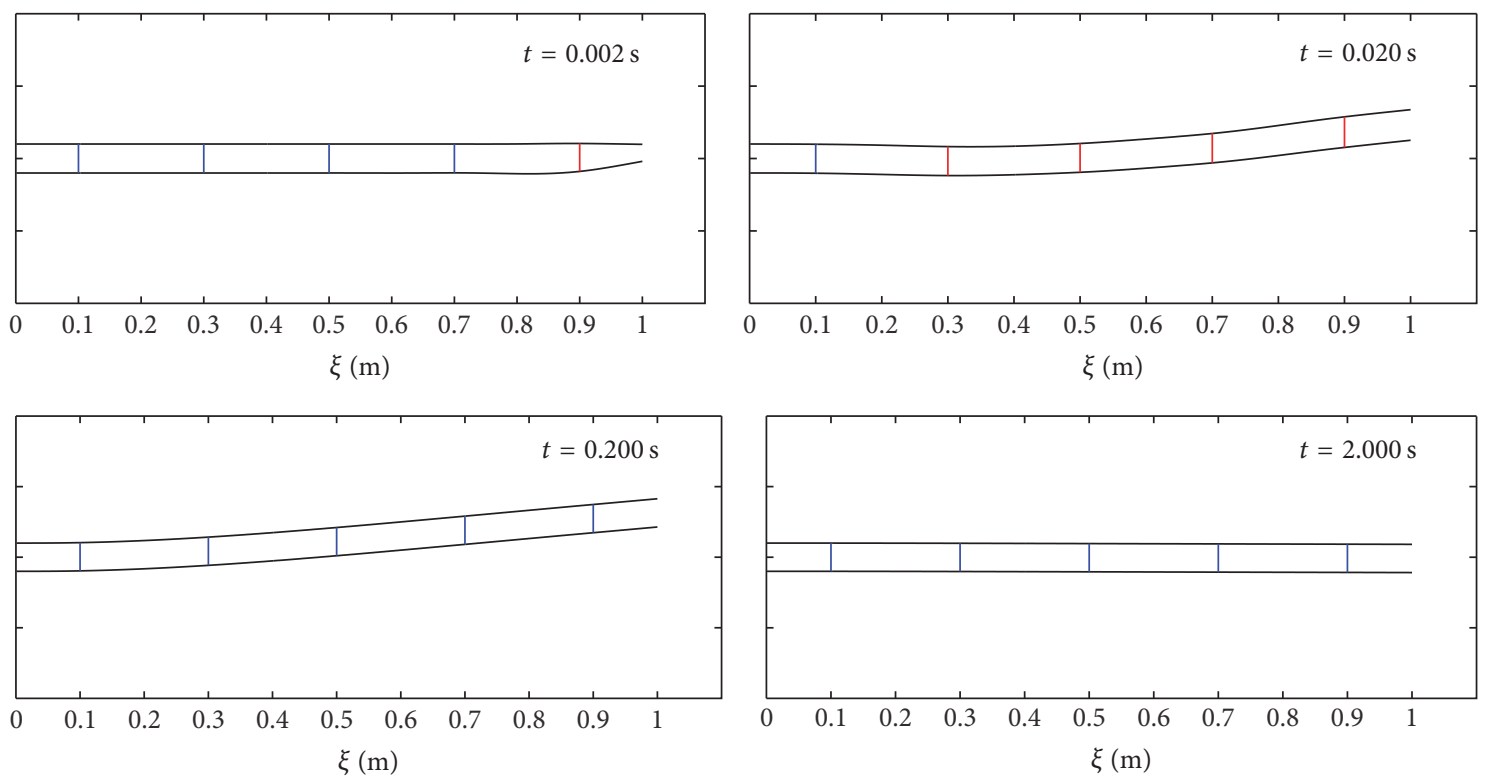

FIGURE 12: Activity of the controlled blocks for the selected time instants in the case of variant 2 . Blue and red colors correspond to $u_{j}=u_{\max }$ and $u_{j}=u_{\text {min }}$, respectively.
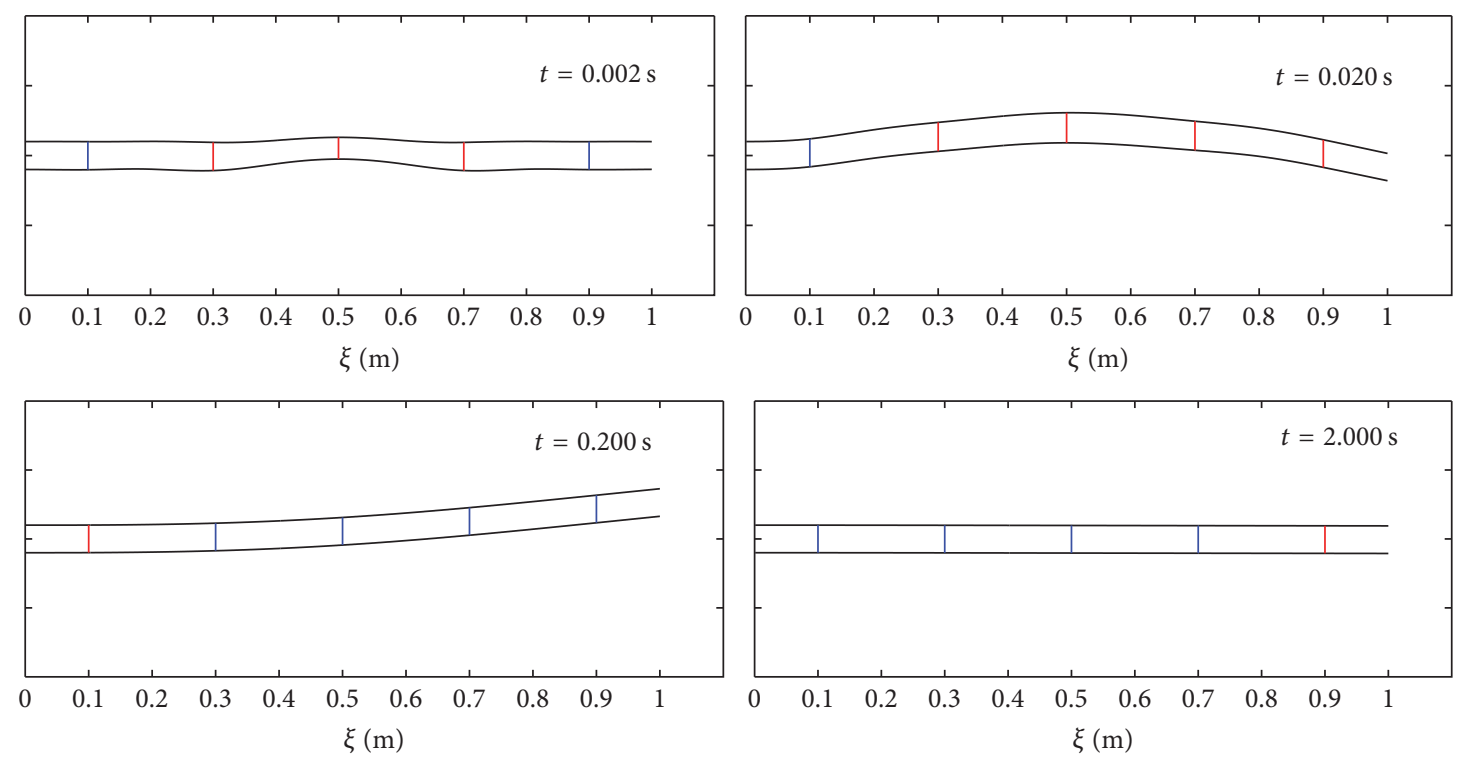

FIGURE 13: Activity of the controlled blocks for the selected time instants in the case of variant 3 . Blue and red colors correspond to $u_{j}=u_{\max }$ and $u_{j}=u_{\text {min }}$, respectively.

operates on two switching modes triggered by a practical and simple implementation state-feedback control law. It provides high rates of the energy dissipation and is robust to a change in the initial condition. For each of the considered scenarios, the closed loop system outperformed the optimal passive cases by over $27 \%$. In this work, the designed controller was suggested for implementation to elastomer based damping blocks that change their mechanical properties due to the magnetic field. The analogous operation, however, can be performed by a number of smart material actuators, and it is applicable to structures as an additional system supporting the existing damping solutions if specific constructional factors, such as the uncertainty of the material properties, nonlinearity, and fail-safe design, are taken into account.

Currently, the authors are preparing an experimental setup to validate the proposed method on a real structure. For the future study, the authors address the problem of designing a distributed structure of the proposed controller. 


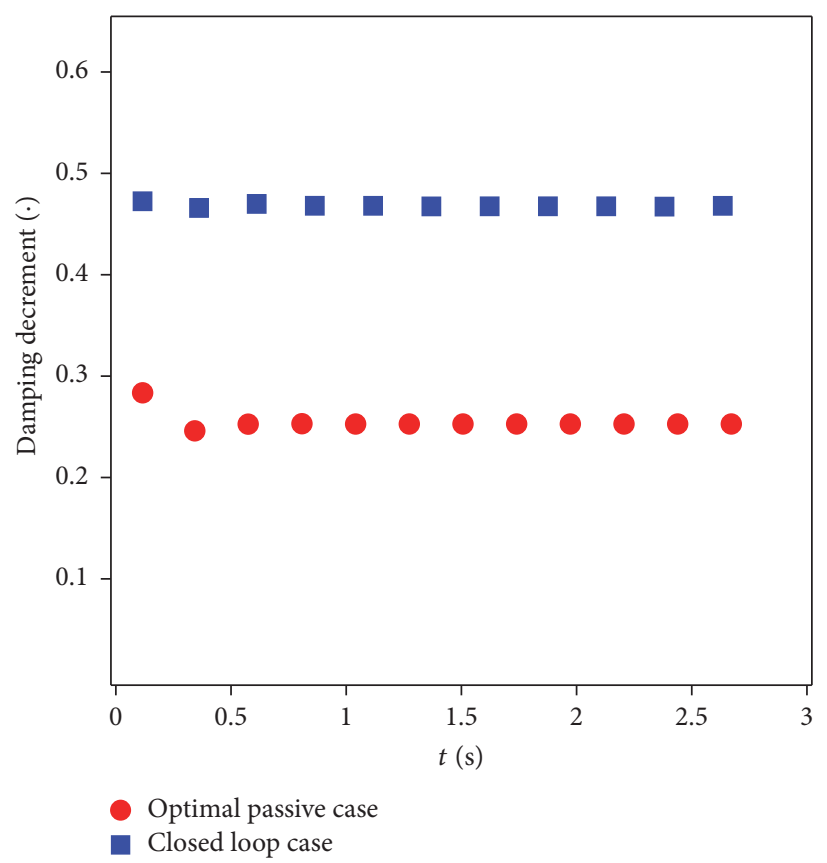

(a)

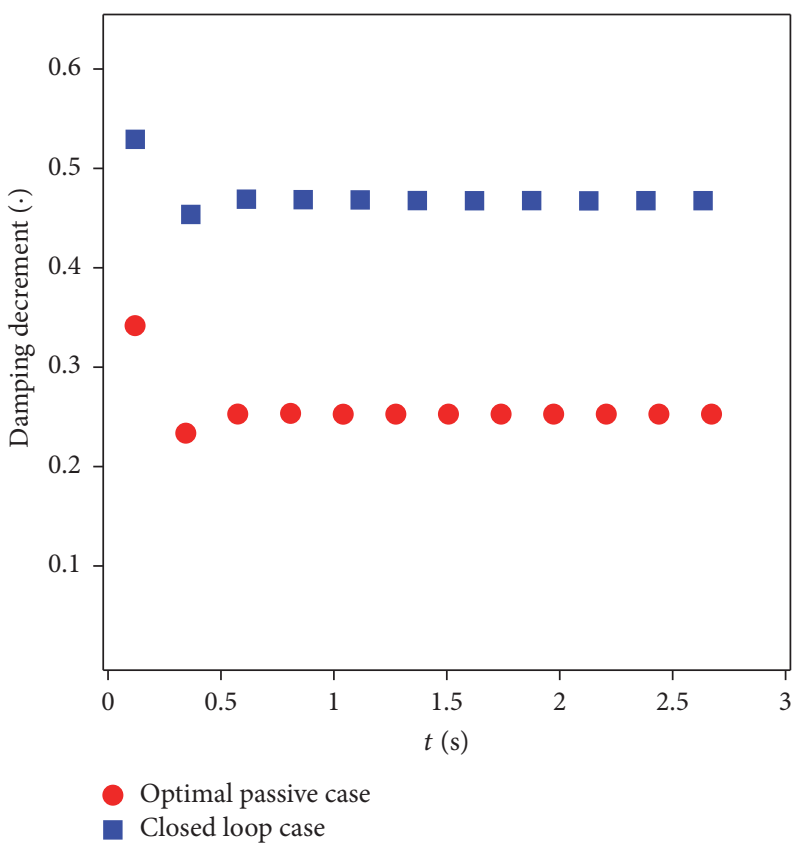

(b)

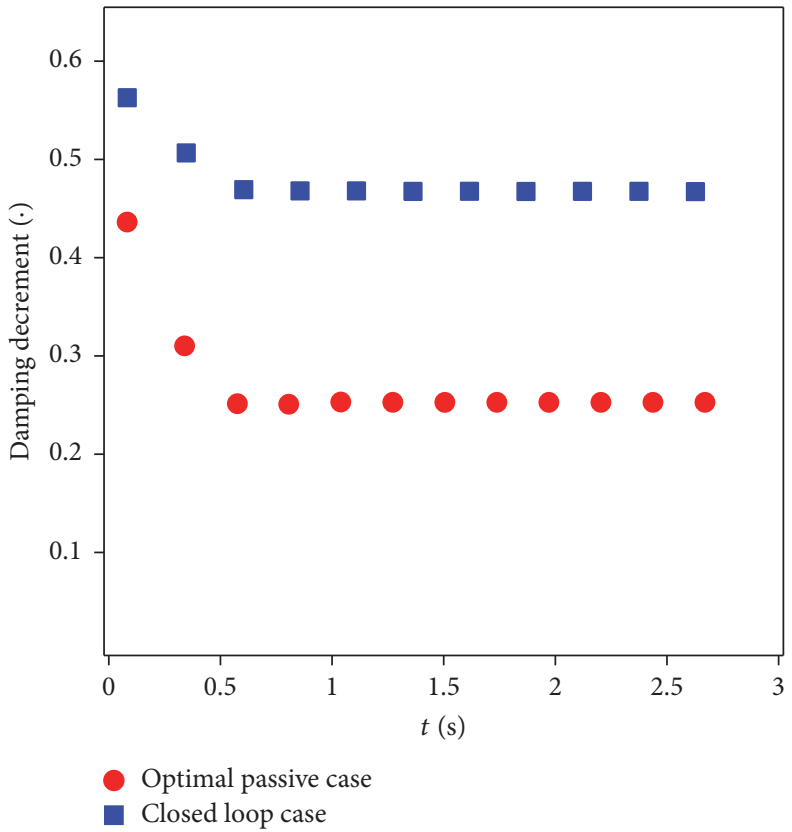

(c)

FiguRE 14: Damping decrements for optimal passive and closed loop cases: (a) variant 1, (b) variant 2, and (c) variant 3.

Relying on a limited amount of state information, it can benefit functionality and robustness.

\section{Competing Interests}

The authors declare that they have no competing interests.

\section{Acknowledgments}

This research has been supported within the frameworks of Projects UMO-2013/11/D/ST8/03437 and UMO-2015/17/B/ ST8/03244 granted by the National Science Centre, which is gratefully acknowledged by the authors. 


\section{References}

[1] D. Pisarski and C. I. Bajer, "Smart suspension system for linear guideways," Journal of Intelligent and Robotic Systems: Theory and Applications, vol. 62, no. 3-4, pp. 451-466, 2011.

[2] J. Onoda, T. Endo, H. Tamaoki, and N. Watanabe, "Vibration suppression by variable-stiffness members," AIAA journal, vol. 29, no. 6, pp. 977-983, 1991.

[3] F. Gandhi and S.-G. Kang, "Beams with controllable flexural stiffness," Smart Materials and Structures, vol. 16, no. 4, pp. 11791184, 2007.

[4] A. Mróz, J. Holnicki-Szulc, and J. Biczyk, "Prestress accumulation-release technique for damping of impact-born vibrations: application to self-deployable structures," Mathematical Problems in Engineering, vol. 2015, Article ID 720236, 9 pages, 2015.

[5] A. Douay and N. Hagood, "Evaluation of optimal variable stiffness feedback control authority stability, feasibility and implementability," in Proceedings of 4th International Conference on Adaptive Structures, pp. 388-404, Cologne, Germany, 1993.

[6] T. Kobs and J. Q. Sun, "A non-linear variable stiffness feedback control with tuning range and rate saturation," Journal of Sound and Vibration, vol. 205, no. 2, pp. 243-249, 1997.

[7] A. Ramaratnam and N. Jalili, "A switched stiffness approach for structural vibration control: theory and real-time implementation," Journal of Sound and Vibration, vol. 291, no. 1-2, pp. 258274, 2006.

[8] D. A. Perkins, J. L. Reed, and E. Havens, "Adaptive wing structures," in Smart Structures and Materials 2004: Industrial and Commercial Applications of Smart Structures Technologies, vol. 5388 of Proceedings of SPIE, pp. 225-233, July 2004.

[9] W. Ostachowicz, K. Majewska, and A. Zak, "Magnetic shape memory alloys for forced vibration control of beam-like structures," Smart Materials and Structures, vol. 16, no. 6, pp. 23882397, 2007.

[10] J. M. Bajkowski, B. Dyniewicz, and C. I. Bajer, "Damping properties of a beam with vacuum-packed granular damper," Journal of Sound and Vibration, vol. 341, pp. 74-85, 2015.

[11] D. Pisarski, C. I. Bajer, B. Dyniewicz, and J. M. Bajkowski, "Vibration control in smart coupled beams subjected to pulse excitations," Journal of Sound and Vibration, vol. 380, pp. 37-50, 2016.

[12] T. Nasu, T. Kobori, M. Takahashi, N. Niwa, and K. Ogasawara, "Active variable stiffness system with non-resonant control," Earthquake Engineering and Structural Dynamics, vol. 30, no. 11, pp. 1597-1614, 2001.

[13] J. N. Yang, J.-H. Kim, and A. K. Agrawal, "Resetting semiactive stiffness damper for seismic response control," Journal of Structural Engineering, vol. 126, no. 12, pp. 1427-1433, 2000.

[14] S. Sahasrabudhe and S. Nagarajaiah, "Effectiveness of variable stiffness systems in base-isolated bridges subjected to near-fault earthquakes: an experimental and analytical study," Journal of Intelligent Material Systems and Structures, vol. 16, no. 9, pp. 743756, 2005.

[15] M. F. Winthrop, W. P. Baker, and R. G. Cobb, "A variable stiffness device selection and design tool for lightly damped structures," Journal of Sound and Vibration, vol. 287, no. 4-5, pp. 667-682, 2005.

[16] B. Dyniewicz, J. M. Bajkowski, and C. I. Bajer, "Semi-active control of a sandwich beam partially filled with magnetorheological elastomer," Mechanical Systems and Signal Processing, vol. 60-61, pp. 695-705, 2015.
[17] T. Szmidt, "Shear deformation damping of a double-beam structure," Journal of Sound and Vibration, vol. 370, pp. 163-175, 2016.

[18] M. Petyt, Introduction to Finite Element Vibration Analysis, Cambridge University Press, 1990.

[19] R. R. Mohler, Bilinear Control Processes, Academic Press, New York, NY, USA, 1973.

[20] D. Pisarski, "Distributed control design for structures subjected to traveling loads," Mathematical Problems in Engineering, vol. 2015, Article ID 206870, 12 pages, 2015.

[21] J. A. Burton and A. S. I. Zinober, "Continuous approximation of variable structure control," International Journal of Systems Science, vol. 17, no. 6, pp. 875-885, 1986. 


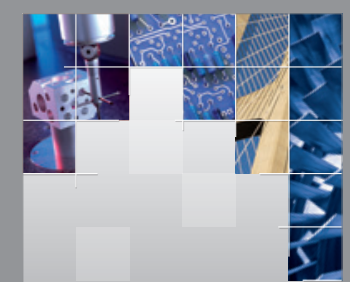

\section{Enfincering}
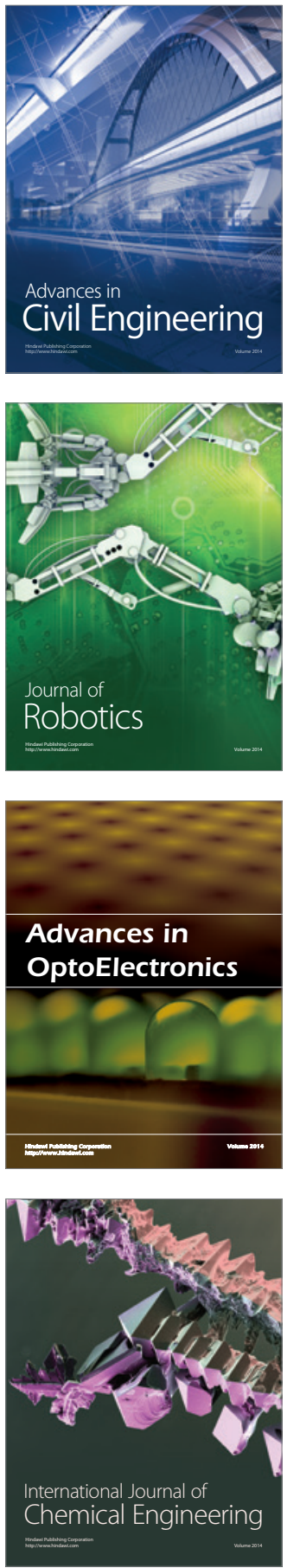

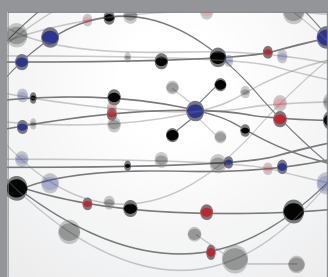

The Scientific World Journal

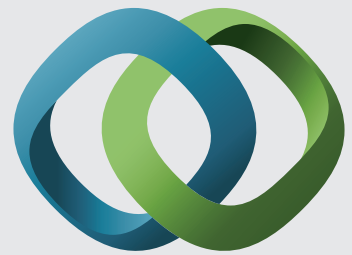

\section{Hindawi}

Submit your manuscripts at

http://www.hindawi.com
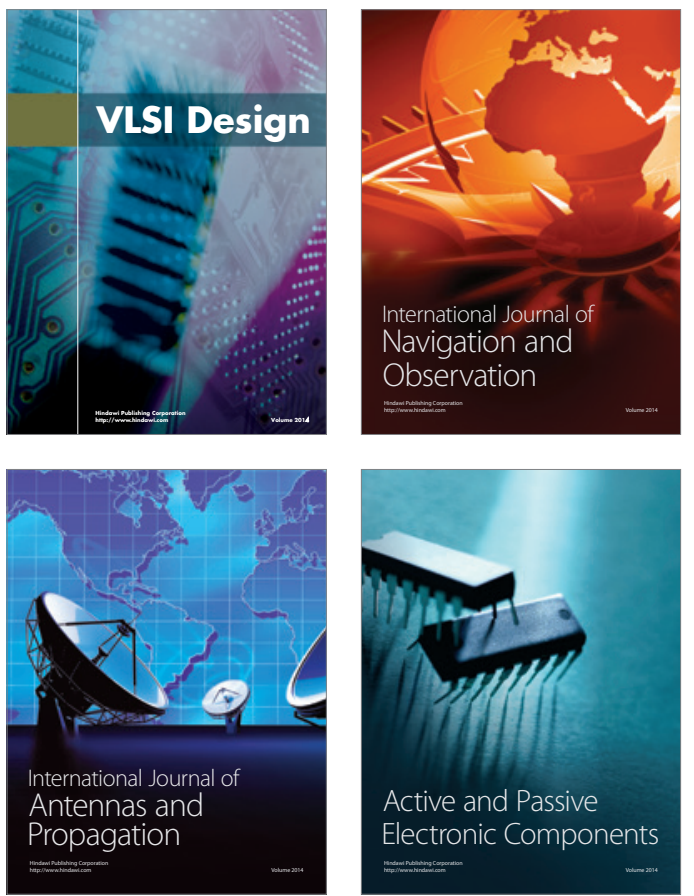
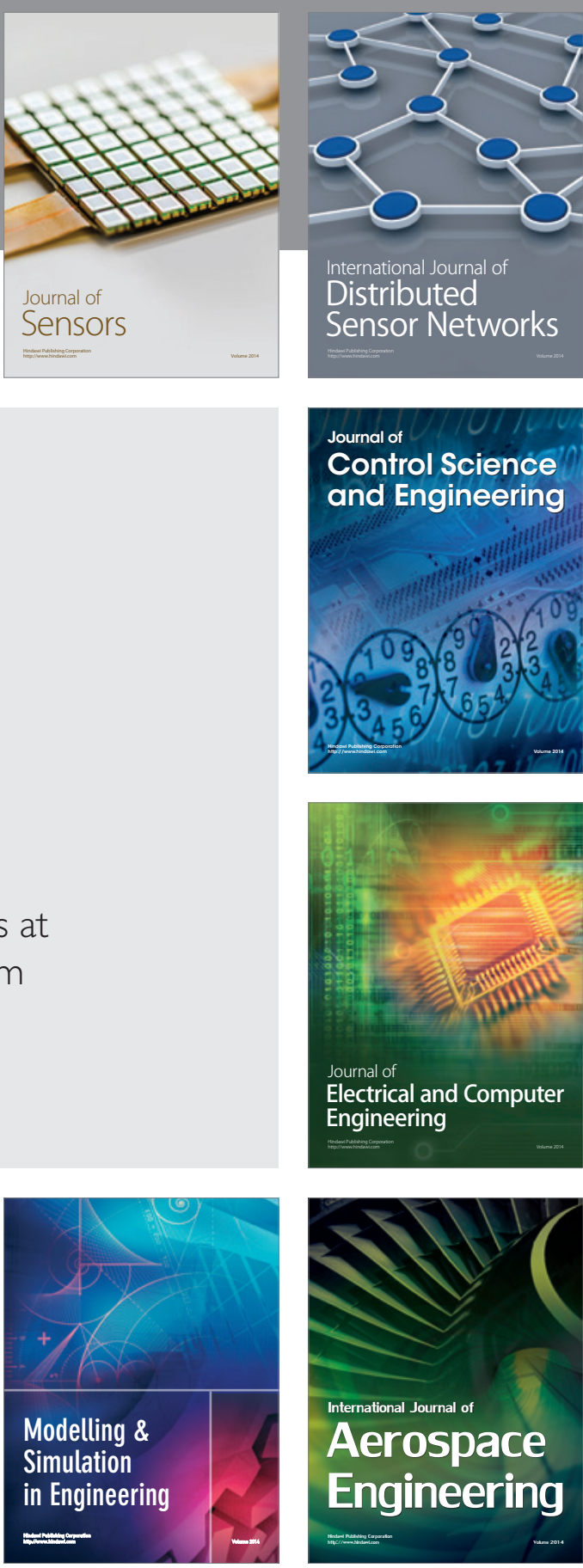

International Journal of

Distributed

Sensor Networks

Journal of

Control Science

and Engineering
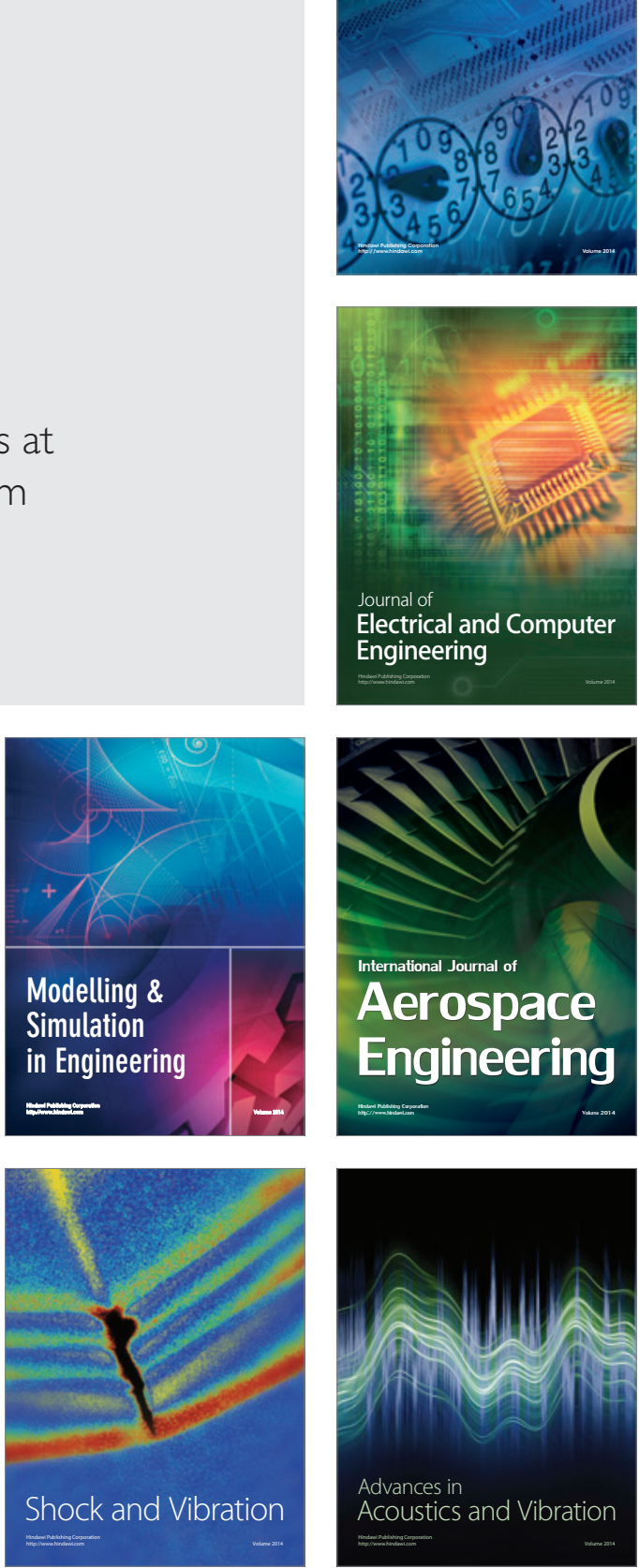